\title{
Sensitivity Analysis of Distributed Parameter Systems
} MASATO KODA AND JOHN H. SEINFELD

\begin{abstract}
A method is developed for the calculation of sensitivity coefficients of general distributed parameter systems to the variation of spatially and temporally varying parameters appearing in the system equations and initial and boundary conditions.
\end{abstract}

\section{INTRODUCTION}

A wide variety of phenomena are described by partial differential equations, so-called distributed parameter systems. In such partial differential equation models it is often desirable to determine the effect of uncertainties in parameters appearing in the equations on their solutions and also, if more than one parameter may vary, which parameters are most influential.

The sensitivity analysis of distributed parameter systems poses several problems that do not arise in the lumped parameter case. First, parameters may occur in the boundary conditions as well as in the differential equations. Second, parameters may vary spatially and/or temporally. Comparatively little work has been done on the distributed parameter sensitivity analysis problem as opposed to the corresponding lumped parameter case. Koda et al. [1] developed methods for the local sensitivity analysis of constant, spatially varying, and temporally varying parameters in parabolic partial differential equations characteristic of reaction and diffusion processes. In the present work we extend that analysis to a general class of distributed systems and apply the results to a problem in one-dimensional heat conduction.

\section{Definition of the Problem}

We consider the class of systems described by

$$
\frac{\partial x}{\partial t}=f\left(x, \frac{\partial x}{\partial z}, \frac{\partial^{2} x}{\partial z^{2}}, u\right) \quad z \in \Omega, t>0
$$

where $\boldsymbol{x}(z, t)$ is the $n$-dimensional state vector and $\boldsymbol{u}(z, t)$ is an $\boldsymbol{m}$-dimensional parameter vector that may, in general, depend on time $t$ and spatial location $z$. For simplicity we consider a scalar spatial variable $z$ defined on domain $\Omega$, a subset of $R_{1}$, with boundaries at $z=0$ and $z=L$. All results to be obtained can be readily extended to several spatial dimensions.

The initial condition to (1) is

$$
\boldsymbol{x}(z, 0)=w(z)
$$

Manuscript received April 25, 1980.

M. Koda is with the Department of Aeronautics, University of Tokyo, Tokyo 113, Japan. J. $\mathrm{H}$. Seinfeld is with the Department of Chemical Engineering, California Institute of Technology, Pasadena, CA 91125. 
The boundary conditions to (1) are taken to fall into either of two categories as follows:

$$
\begin{aligned}
\text { 1) } \frac{\partial x_{i}}{\partial z} & =g_{i}(x, v(t)) \quad z=0 \\
\frac{\partial x_{i}}{\partial z} & =h_{i}(x, y(t)) \quad z=L \quad i=1,2, \ldots, s \\
\text { 2) } x_{i}(0, t) & =v_{i}(t) \\
x_{i}(L, t) & =y_{i}(t) \quad i=s+1, \ldots, n .
\end{aligned}
$$

Thus, $s$ of the state variables are assumed to obey conditions (3) and (4), and $n-s$ to obey (5) and (6). This specification includes virtually all systems of practical interest. $v(t)$ and $y(t)$ are $n$-dimensional parameter vectors, respectively, assumed for generality to be functions of time $t$.

We have defined a general distributed parameter system, the state, $\boldsymbol{x}(z, t)$, of which depends on the values of parameters, $\boldsymbol{u}(z, t), \boldsymbol{w}(z), \boldsymbol{v}(t)$, and $y(t)$. A local sensitivity analysis seeks to determine the effect of small perturbations of these parameters about their nominal values on $x(z, t)$. Whereas for constant parameters the partial derivatives, $\partial x_{i}(z, t) / \partial u_{j}$, contain the desired information, for spatially and temporally varying parameters the quantities of direct interest are the functional derivatives, $\delta x_{i}\left(z^{\prime}, t^{\prime}\right) / \delta u_{j}(z, t), \quad \delta x_{i}\left(z^{\prime}, t\right) / \delta w_{j}(z), \delta x_{i}\left(z, t^{\prime}\right) / \delta v_{j}(t)$, and $\delta x_{i}\left(z, t^{\prime}\right) / \delta y_{j}(t)$. The functional derivatives are defined such that the change in $x_{i}(z, t)$ as a result of perturbations in the parameters $u_{j}(z, t)$, $w_{j}(z), v_{j}(t)$, and $y_{j}(t)$ are

$$
\begin{aligned}
& \delta x_{i}\left(z^{\prime}, t^{\prime}\right)=\int_{0}^{t_{f}} \int_{0}^{L} \sum_{j=1}^{m} \frac{\delta x_{i}\left(z^{\prime}, t^{\prime}\right)}{\delta u_{j}(z, t)} \delta u_{j}(z, t) d z d t \\
& \delta x_{i}\left(z^{\prime}, t\right)=\int_{0}^{L} \sum_{j=1}^{n} \frac{\delta x_{i}\left(z^{\prime}, t\right)}{\delta w_{j}(z)} \delta w_{j}(z) d z \\
& \delta x_{i}\left(z, t^{\prime}\right)=\int_{0}^{t_{j}} \sum_{j=1}^{n} \frac{\delta x_{i}\left(z, t^{\prime}\right)}{\delta v_{j}(t)} \delta v_{j}(t) d t \\
& \delta x_{i}\left(z, t^{\prime}\right)=\int_{0}^{t_{f}} \sum_{j=1}^{n} \frac{\delta x_{i}\left(z, t^{\prime}\right)}{\delta y_{j}(t)} \delta y_{j}(t) d t .
\end{aligned}
$$

Thus, for specific values of $z^{\prime}$ and $t^{\prime}, \delta x_{i}\left(z^{\prime}, t^{\prime}\right) / \delta u_{j}(z, t)$ can be interpreted as the sensitivity of $x_{i}$ at $z^{\prime}$ and $t^{\prime}$ to variations in $u_{j}$ at $z$ and $t$. Likewise, for a specific value of $z^{\prime}, \delta x_{i}\left(z^{\prime}, t\right) / \delta w_{j}(z)$ is the sensitivity of $x_{i}$ at $z^{\prime}$ and $t$ to variations in $w_{j}$ at $z$. Similar interpretations can be ascribed to the functional derivatives appearing in (9) and (10).

Our object is to obtain expressions for the functional derivative sensitivity coefficients appearing in (7)-(10). Results are presented in the next section that provide the means to calculate the desired sensitivity coefficients. Then in the section following, the results are applied to a problem of one-dimensional heat conduction.

\section{Functional Derivative Sensitivity Coefficients}

The expressions for the functional derivative sensitivity coefficients can be obtained from distributed parameter optimal control theory as applied to the system (1)-(6) [2], [3]. Assume for the moment that $\boldsymbol{u}, \boldsymbol{w}, \boldsymbol{v}$, and $y$ are control variables and that we have the general performance index

$$
\begin{aligned}
I[\boldsymbol{u}, \boldsymbol{w}, \boldsymbol{v}, \boldsymbol{y}]= & \int_{0}^{L} G_{1}\left(x\left(z, t_{f}\right), w(z)\right) d z \\
& +\int_{0}^{t_{f}} G_{2}(x(L, t), x(0, t), v(t), y(t)) d t \\
& +\int_{0}^{t_{f}} \int_{0}^{L} G\left(x, \frac{\partial x}{\partial z}, \frac{\partial^{2} x}{\partial z^{2}}, u(z, t)\right) d z d t
\end{aligned}
$$

Necessary conditions for optimality can be derived formally by considering the effect of perturbations about the nominal values $\overline{\boldsymbol{u}}, \overline{\boldsymbol{w}}, \overline{\boldsymbol{v}}$, and $\bar{y}$ on I. It is straightforward to show that if Hamiltonians are defined as

$$
\begin{gathered}
H=G+\lambda^{T_{f}} \\
H_{1}=G_{1}+\lambda^{T_{w}}
\end{gathered}
$$

$$
\begin{aligned}
& H_{2}=G_{2}-\left.\sum_{j=1}^{s} \frac{\partial H}{\partial x_{j_{z z}}}\right|_{z=0} g_{j} \\
& H_{3}=G_{2}+\left.\sum_{j=1}^{s} \frac{\partial H}{\partial x_{j_{z z}}}\right|_{z=0} h_{j},
\end{aligned}
$$

the first-order variation in $I$ is given by (see, for example, [3])

$$
\begin{aligned}
\delta I= & \int_{0}^{t_{f}} \int_{0}^{L} \sum_{j=1}^{m} \frac{\partial H}{\partial u_{j}} \delta u_{j} d z d t+\int_{0}^{L} \sum_{j=1}^{n} \frac{\partial H_{1}}{\partial w_{j}} \delta w_{j} d z \\
& +\int_{0}^{t_{f}}\left\{\sum_{j=1}^{s}\left(\frac{\partial H_{2}}{\partial v_{j}} \delta v_{j}+\frac{\partial H_{3}}{\partial y_{j}} \delta y_{j}\right)\right. \\
& +\sum_{j=s+1}^{n}\left[\frac{\partial G_{2}}{\partial v_{j}}-\frac{\partial H}{\partial x_{j_{z}}}+\frac{\partial}{\partial z}\left(\frac{\partial H}{\partial x_{j_{z z}}}\right)\right] \delta v_{j} \\
& \left.+\sum_{j=s+1}^{n}\left[\frac{\partial G_{2}}{\partial y_{j}}+\frac{\partial H}{\partial x_{j_{z}}}-\frac{\partial}{\partial z}\left(\frac{\partial H}{\partial x_{j_{z z}}}\right)\right] \delta y_{j}\right\} d t
\end{aligned}
$$

where the $n$-dimensional adjoint vector $\lambda(z, t)$ is governed by

$$
\frac{\partial \lambda^{T}}{\partial t}=-\frac{\partial H}{\partial x}+\frac{\partial}{\partial z}\left(\frac{\partial H}{\partial x_{z}}\right)-\frac{\partial^{2}}{\partial z^{2}}\left(\frac{\partial H}{\partial x_{z z}}\right)
$$

subject to the terminal condition

$$
\lambda^{T}\left(z, t_{f}\right)=\frac{\partial G_{1}}{\partial x\left(z, t_{f}\right)}
$$

and boundary conditions

$$
\begin{gathered}
\frac{\partial H_{2}}{\partial x_{j}(0, t)}-\frac{\partial H}{\partial x_{j_{z}}}+\frac{\partial}{\partial z}\left(\frac{\partial H}{\partial x_{j_{z z}}}\right)=0 \quad z=0 \\
\frac{\partial H_{3}}{\partial x_{j}(L, t)}+\frac{\partial H}{\partial x_{j_{z}}}-\frac{\partial}{\partial z}\left(\frac{\partial H}{\partial z_{j_{z z}}}\right)=0 \quad z=L \quad j=1,2, \cdots, s \\
\frac{\partial H}{\partial x_{j_{z z}}}=0 \quad z=0, L \quad j=s+1, \cdots, n .
\end{gathered}
$$

We note that if the system (1) is not second-order in some of the states, $x_{q}(z, t)$, then in the original boundary conditions for the system, these states would only be specified at one of the two boundaries, say $z=0$. The boundary condition on the adjoint variables $\lambda_{q}(z, t)$ corresponding to such states is then

$$
\frac{\partial G_{2}}{\partial x_{q}(L, t)}+\frac{\partial H}{\partial x_{q_{z}}}=0 .
$$

The above results may be applied directly to determining the desired functional derivative sensitivity coefficients. If we let $G=G_{2}=0$ and

$$
G_{1}\left(x\left(z, t_{f}\right), w(z)\right)=x_{r}\left(z, t_{f}\right) \delta\left(z-z^{\prime}\right),
$$

then

$$
I_{r}=x_{r}\left(z^{\prime}, t_{f}\right)
$$

where $r$ indicates any of the $n$ states of interest. In this case, the Hamiltonians become

$$
\begin{aligned}
H & =\lambda^{T} f \\
H_{1} & =G_{1}+\lambda^{T} w \\
H_{2} & =-\left.\sum_{j=1}^{s} \frac{\partial H}{\partial x_{j_{z z}}}\right|_{z=0} g_{j} \\
H_{3} & =\left.\sum_{j=1}^{s} \frac{\partial H}{\partial x_{j_{z z}}}\right|_{z=L} h_{j} .
\end{aligned}
$$


The adjoint equations become

$$
\begin{aligned}
\frac{\partial \lambda_{i}}{\partial t}=-\sum_{j=1}^{n} \lambda_{j} \frac{\partial f_{j}}{\partial x_{i}}+\sum_{j=1}^{n} \frac{\partial}{\partial z} & \left(\lambda_{j} \frac{\partial f_{j}}{\partial x_{i_{z}}}\right) \\
& -\sum_{j=1}^{n} \frac{\partial^{2}}{\partial z^{2}}\left(\lambda_{j} \frac{\partial f_{j}}{\partial x_{i_{z z}}}\right) \quad i=1, \cdots, n
\end{aligned}
$$

subject to the terminal condition

$$
\lambda_{i}\left(z, t_{f}\right)= \begin{cases}\delta\left(z-z^{\prime}\right) & i=r \\ 0 & i \neq r\end{cases}
$$

and boundary conditions

$$
\begin{aligned}
& -\sum_{k=1}^{s} \sum_{j=1}^{n} \lambda_{j}(0, t)\left(\frac{\partial f_{i}}{\partial x_{k_{z z}}}\right)_{z=0} \frac{\partial g_{k}}{\partial x_{i}(0, t)}-\sum_{k=1}^{n} \lambda_{k}(0, t)\left(\frac{\partial f_{k}}{\partial x_{i_{z}}}\right)_{z=0} \\
& +\sum_{k=1}^{n}\left[\left(\frac{\partial \lambda_{k}}{\partial z}\right)_{z=0}\left(\frac{\partial f_{k}}{\partial x_{i z z}}\right)_{z=0}+\lambda_{k}(0, t) \frac{\partial}{\partial z}\left(\frac{\partial f_{k}}{\partial x_{i z z}}\right)_{z=0}\right]=0 \\
& \sum_{k=1}^{s} \sum_{j=1}^{n} \lambda_{j}(L, t)\left(\frac{\partial f_{i}}{\partial x_{k z z}}\right)_{z=L} \frac{\partial h_{k}}{\partial x_{i}(L, t)}+\sum_{k=1}^{n} \lambda_{k}(L, t)\left(\frac{\partial f_{k}}{\partial x_{i z}}\right)_{z=L} \\
& -\sum_{k=1}^{n}\left[\left(\frac{\partial \lambda_{k}}{\partial z}\right)_{z=L}\left(\frac{\partial f_{k}}{\partial x_{i_{z z}}}\right)_{z=L}+\lambda_{k}(L, t)\left(\frac{\partial f_{k}}{\partial x_{i_{z z}}}\right)_{z=L}\right]=0 \\
& i=1,2, \cdots, s \\
& \sum_{k=1}^{n} \lambda_{k}(0, t)\left(\frac{\partial f_{k}}{\partial x_{i_{z z}}}\right)_{z=0}=0 \\
& \sum_{k=1}^{n} \lambda_{k}(L, t)\left(\frac{\partial f_{k}}{\partial x_{i_{z z}}}\right)_{z=L}=0 \quad i=s+1, \cdots, n \text {. }
\end{aligned}
$$

The perturbation $\delta I_{r}=\delta x_{r}\left(z^{\prime}, t_{f}\right)$ is then given by

$$
\begin{aligned}
\delta I_{r}= & \int_{0}^{t_{f}} \int_{0}^{L} \sum_{i=1}^{m} \sum_{k=1}^{n} \lambda_{k}(z, t) \frac{\partial f_{k}}{\partial u_{i}} \delta u_{i}(z, t) d z d t \\
& +\int_{0}^{L} \sum_{i=1}^{n} \lambda_{i}(z, 0) \delta w_{i}(z) d z \\
& -\int_{0}^{t_{f}} \sum_{i=1}^{s} \sum_{k=1}^{s} \sum_{j=1}^{n} \lambda_{j}(0, t)\left(\frac{\partial f_{j}}{\partial x_{k_{z z}}}\right)_{z=0} \frac{\partial g_{k}}{\partial v_{i}} \delta v_{i}(t) d t \\
& +\int_{0}^{t_{f}} \sum_{i=1}^{s} \sum_{k=1}^{s} \sum_{j=1}^{n} \lambda_{j}(L, t)\left(\frac{\partial f_{j}}{\partial x_{k_{z z}}}\right)_{z=L} \frac{\partial h_{k}}{\partial y_{i}} \delta y_{i}(t) d t \\
& +\int_{0}^{t_{f}} \sum_{i=s+1}^{n} \sum_{k=1}^{n}\left[-\lambda_{k}(0, t)\left(\frac{\partial f_{k}}{\partial x_{i_{z}}}\right)_{z=0}+\left(\frac{\partial \lambda_{k}}{\partial z}\right)_{z=0}\left(\frac{\partial f_{k}}{\partial x_{i_{z z}}}\right)_{z=0}\right. \\
& \left.+\left.\lambda_{k}(0, t) \frac{\partial}{\partial z}\left(\frac{\partial f_{k}}{\partial x_{i_{z z}}}\right)\right|_{z=0}\right] \delta v_{i}(t) d t \quad(29) \\
& +\int_{0}^{t_{f}} \sum_{i=s+1}^{n} \sum_{k=1}^{n}\left[\lambda_{k}(L, t)\left(\frac{\partial f_{k}}{\partial x_{i_{z}}}\right)_{z=L}-\left(\frac{\partial \lambda_{k}}{\partial z}\right)_{z=L}\left(\frac{\partial f_{k}}{\partial x_{i_{z z}}}\right)_{z=L}\right. \\
& \left.-\left.\lambda_{k}(L, t) \frac{\partial}{\partial z}\left(\frac{\partial f_{k}}{\partial x_{i_{z z}}}\right)\right|_{z=L}\right] \delta y_{i}(t) d t .
\end{aligned}
$$

Recalling (7)-(10), the desired functional derivative sensitivity coefficients are obtained directly from (29) as

$$
\begin{aligned}
& \frac{\delta x_{r}\left(z^{\prime}, t_{f}\right)}{\delta u_{i}(z, t)}=\sum_{k=1}^{n} \lambda_{k}(z, t) \frac{\partial f_{k}}{\partial u_{i}} \\
& \frac{\delta x_{r}\left(z^{\prime}, t_{f}\right)}{\delta w_{i}(z)}=\lambda_{i}(z, 0)
\end{aligned}
$$

$$
\begin{aligned}
& \frac{\delta x_{r}\left(z^{\prime}, t_{f}\right)}{\delta v_{i}(t)}=-\sum_{k=1}^{s} \sum_{j=1}^{n} \lambda_{j}(0, t)\left(\frac{\partial f_{j}}{\partial x_{k_{z z}}}\right)_{z=0} \frac{\partial g_{k}}{\partial v_{i}} \\
& \frac{\delta x_{r}\left(z^{\prime}, t_{f}\right)}{\delta y_{i}(t)}=\sum_{k=1}^{s} \sum_{j=1}^{n} \lambda_{j}(L, t)\left(\frac{\partial f_{j}}{\partial x_{k_{x z}}}\right)_{z=L} \frac{\partial h_{k}}{\partial y_{i}} \quad i=1,2, \cdots, s \quad(3, \cdots, s
\end{aligned}
$$

$$
\begin{aligned}
\frac{\delta x_{r}\left(z^{\prime}, t_{f}\right)}{\delta v_{i}(t)}=\sum_{k=1}^{n}\left[-\lambda_{k}(0, t)\left(\frac{\partial f_{k}}{\partial x_{i_{z}}}\right)_{z=0}+\left(\frac{\partial \lambda_{k}}{\partial z}\right)_{z=0}\left(\frac{\partial f_{k}}{\partial x_{i_{z z}}}\right)_{z=0}\right. \\
\left.+\left.\lambda_{k}(0, t) \frac{\partial}{\partial z}\left(\frac{\partial f_{k}}{\partial x_{i_{z z}}}\right)\right|_{z=0}\right] \quad i=s+1, \cdots, n \quad(34) \\
\frac{\delta x_{r}\left(z^{\prime}, t_{f}\right)}{\delta y_{i}(t)}=\sum_{k=1}^{n}\left[\lambda_{k}(L, t)\left(\frac{\partial f_{k}}{\partial x_{i_{z}}}\right)_{z=L}-\left(\frac{\partial \lambda_{k}}{\partial z}\right)_{z=L}\left(\frac{\partial f_{k}}{\partial x_{i_{z z}}}\right)_{z=L}\right. \\
\left.-\left.\lambda_{k}(L, t) \frac{\partial}{\partial z}\left(\frac{\partial f_{k}}{\partial x_{i_{z z}}}\right)\right|_{z=L}\right] \quad i=s+1, \cdots, n . \quad(35)
\end{aligned}
$$

\section{Sensitivity Analysis of One-Dimensional Heat CONDUCTION}

The dimensionless temperature $x(z, t)$ in a rod occupying the domain $z \in[0,1]$ is governed by the heat equation

$$
\frac{\partial x}{\partial t}=\frac{\partial^{2} x}{\partial z^{2}}
$$

For a given initial condition, $x(z, 0)=w(z)$, we wish to examine the sensitivity of $x(z, t)$ to parameters appearing in the boundary conditions at $z=0$ and $z=1$. We consider the two cases introduced earlier. In case 1) convective heat transfer occurs from both the $z=0$ and $z=1$ boundaries with heat transfer coefficients $v(t)$ and $y(t)$ as follows:

$$
\text { 1) } \begin{array}{ll}
\frac{\partial x}{\partial z}=v(t) x & z=0 \\
\frac{\partial x}{\partial z}=y(t) x & z=1 .
\end{array}
$$

In case 2) the temperatures at $z=0$ and $z=1$ are maintained at the values $v(t)$ and $y(t)$ as follows:

$$
\text { 2) } \begin{aligned}
x(0, t) & =v(t) \\
x(1, t) & =y(t)
\end{aligned}
$$

We wish to apply the general sensitivity analysis theory developed above to determine $\delta x\left(z, t_{f}\right) / \delta v(t)$ and $\delta x\left(z, t_{f}\right) / \delta y(t)$ in cases 1) and 2 ). In each case the nominal values of $v$ and $y$ are chosen to be constants $\bar{v}$ and $\bar{y}$, respectively.

Case 1): We seek to determine the sensitivity of $x(z, t)$ to perturbations of $v(t)$ and $y(t)$ about their nominal values of $\bar{v}=1, \bar{y}=-1$ when the $z=0,1$ boundary conditions are (37) and (38). The initial condition selected is $x(z, 0)=a\left(\cos \alpha_{1} z+\left(\sin \alpha_{1} z\right) / \alpha_{1}\right)$, where $a$ is a constant and $\alpha_{1}$ satisfies $\tan \alpha_{1}=2 \alpha_{1} /\left(\alpha_{1}^{2}-1\right)$. The adjoint system in this case is

$$
\begin{aligned}
\frac{\partial \lambda}{\partial t} & =-\frac{\partial^{2} \lambda}{\partial z^{2}} \\
\lambda\left(z, t_{f}\right) & =\delta\left(z-z^{\prime}\right) \\
\frac{\partial \lambda}{\partial z} & =\lambda \quad z=0 \\
\frac{\partial \lambda}{\partial z} & =-\lambda \quad z=1 .
\end{aligned}
$$




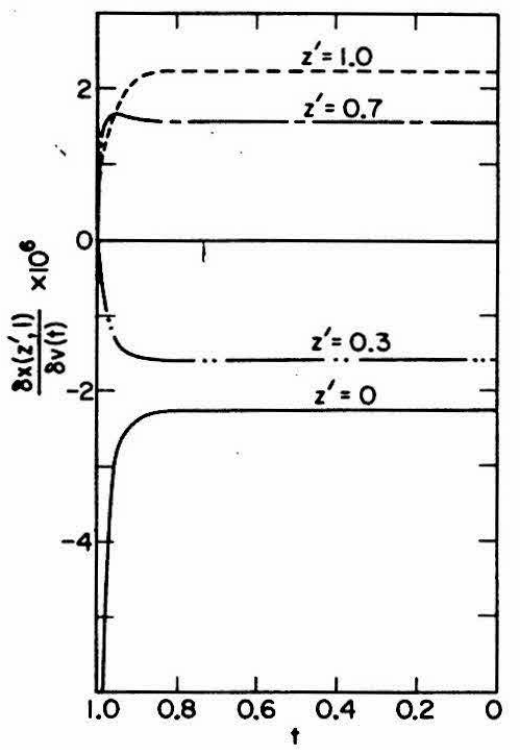

Fig. 1. Functional derivative sensitivity coefficient $\delta x\left(z^{\prime}, 1\right) / \delta v(t)$ of the state at $t_{f}=1$ as a function of $t$ for four values of $z^{\prime}$. Case 1 ) boundary conditions.

The solutions for $x(z, t)$ and $\lambda(z, t)$ are

$$
\begin{aligned}
x(z, t)= & a\left(\cos \alpha_{1} z+\frac{1}{\alpha_{1}} \sin \alpha_{1} z\right) e^{-\alpha_{1}^{2} t} \\
\lambda(z, t)= & 2 \sum_{n=1}^{\infty} \frac{e^{-\alpha_{n}^{2}(t,-t)}}{\alpha_{n}^{2}+3}\left(\alpha_{n} \cos \alpha_{n} z^{\prime}+\sin \alpha_{n} z^{\prime}\right) \\
& \cdot\left(\alpha_{n} \cos \alpha_{n} z+\sin \alpha_{n} z\right)
\end{aligned}
$$

where $\alpha_{n}$ is the $n$th positive root of $\tan \alpha_{n}=2 \alpha_{n} /\left(\alpha_{n}^{2}-1\right)$.

The desired functional derivative sensitivity coefficients are

$$
\begin{aligned}
\frac{\delta x\left(z^{\prime}, t_{f}\right)}{\delta v(t)}= & -\lambda(0, t) x(0, t) \\
= & -2 a \sum_{n=1}^{\infty} \frac{\alpha_{n}}{\alpha_{n}^{2}+3} \exp \left[-\alpha_{n}^{2} t_{f}-\left(\alpha_{1}^{2}-\alpha_{n}^{2}\right) t\right] \\
& \cdot\left(\alpha_{n} \cos \alpha_{n} z^{\prime}+\sin \alpha_{n} z^{\prime}\right) \\
\frac{\delta x\left(z^{\prime}, t_{f}\right)}{\delta y(t)}= & \lambda(1, t) x(1, t)=\frac{\delta x\left(1-z^{\prime}, t_{f}\right)}{\delta v(t)} .
\end{aligned}
$$

On the basis of (48) we see that it is only necessary to examine the sensitivity of $x\left(z^{\prime}, t_{f}\right)$ to one of the parameters, say $v(t)$.

Case 2): In this case we select the nominal values $\bar{v}=\bar{y}=a$ for use with boundary conditions (39) and (40) and the initial condition $x(z, 0)=a(1$ $+\sin \pi z$ ), where $a>0$. The adjoint system is

$$
\begin{aligned}
\frac{\partial \lambda}{\partial t} & =-\frac{\partial^{2} \lambda}{\partial z^{2}} \\
\lambda(0, t) & =\lambda(1, t)=0 \\
\lambda\left(z, t_{f}\right) & =\delta\left(z-z^{\prime}\right) .
\end{aligned}
$$

The solutions for $x(z, t)$ and $\lambda(z, t)$ are

$$
\begin{aligned}
& x(z, t)=a\left[1+\sin (\pi z) e^{-\pi^{2} t}\right] \\
& \lambda(z, t)=2 \sum_{n=1}^{\infty} \sin \left(n \pi z^{\prime}\right) \sin (n \pi z) e^{-(n \pi)^{2}(t,-t)}
\end{aligned}
$$

and the desired functional derivatives are

$$
\begin{aligned}
\frac{\delta x\left(z^{\prime}, t_{f}\right)}{\delta v(t)} & =\left(\frac{\partial \lambda}{\partial z}\right)_{z=0} \\
& =2 \pi \sum_{n=1}^{\infty} n \sin \left(n \pi z^{\prime}\right) e^{-(n \pi)^{2}\left(t_{f}-t\right)} \\
\frac{\delta x\left(z^{\prime}, t_{f}\right)}{\delta y(t)} & =-\left(\frac{\partial \lambda}{\partial z}\right)_{z=1} \\
& =\frac{\delta x\left(1-z^{\prime}, t_{f}\right)}{\delta v(t)} .
\end{aligned}
$$

As in case 1), we need examine only one of the sensitivities, say that to $v(t)$.

Numerical Results: We now evaluate numerically the functional derivative sensitivity coefficients for cases 1) and 2). For this purpose we set $t_{f}=1$ and $a=1$.

Case 1): $\delta x\left(z^{\prime}, 1\right) / \delta v(t)$ is shown in Fig. 1 for $z^{\prime}=0,0.3,0.7$, and 1.0. These curves show the sensitivity of the temperature at $t=1$ at the four locations to variations in $v(t)$ at all past times $t$. For this reason the time axis is reversed in Fig. 1. We note that at $z^{\prime}=0, \delta x\left(z^{\prime}, 1\right) / \delta v(t) \rightarrow-\infty$. This behavior is to be expected as a result of the $z=0$ boundary condition (37). For $z^{\prime}>0, \delta x\left(z^{\prime}, 1\right) / \delta v(t)=0$ at $t=1$ and converges to a constant value as $t$ decreases toward zero. Throughout all past times $\delta x\left(z^{\prime}, 1\right) / \delta v(t)<0$ for $z^{\prime}<0.5$ and $>0$ for $z^{\prime}>0.5$, indicating that an increase in $v(t)$ at any time between 0 and 1 tends to decrease $x\left(z^{\prime}, 1\right)$ for $z^{\prime}<0.5$ and tends to increase $x\left(z^{\prime}, 1\right)$ for $z^{\prime}>0.5$. As expected, $x(0,1)$ is most sensitive to any changes in $v(t)$. The spatial distribution of $\delta x\left(z^{\prime}, 1\right) / \delta v(t)$ is shown in Fig. 2 for $t=0.5$ and $0.9 . \delta x\left(z^{\prime}, 1\right) / \delta v(t)$ achieves its largest value, as we know, at $z^{\prime}=0$ and reverses sign at $z^{\prime}=0.5$.

Case 2): $\delta x\left(z^{\prime}, 1\right) / \delta v(t)$ is shown in Fig. 3 for $z^{\prime}=0.3,0.5$, and 0.7. As $z^{\prime} \rightarrow 0, \delta x\left(z^{\prime}, 1\right) / \delta v(t) \rightarrow \infty$, due to the boundary condition (39). The value of $\delta x\left(z^{\prime}, 1\right) / \delta v(t)$ decreases exponentially as $t \rightarrow 0$. For all $t, \delta x\left(z^{\prime}, 1\right) / \delta v(t)>0$, indicating that an increase in $v(t)$ increases $x\left(z^{\prime}, 1\right)$ for any $z^{\prime}$, as expected from (39). Fig. 4 shows $\delta x\left(z^{\prime}, 1\right) / \delta v(t)$ as a function of $z^{\prime}$ for $t=0.9,0.8$, and 0.5 . The maximum value occurs at $z^{\prime}=0.5$ for $t<0.8$ because $x$ assumes its largest value at $z=0.5$.

\section{Conclusions}

Sensitivity coefficients for spatially and temporally varying parameters in partial differential equations are derived from techniques of distributed parameter optimal control theory. The theory is applied to a problem based on the heat equation in which the sensitivity of the state to variations in boundary parameters is calculated. 


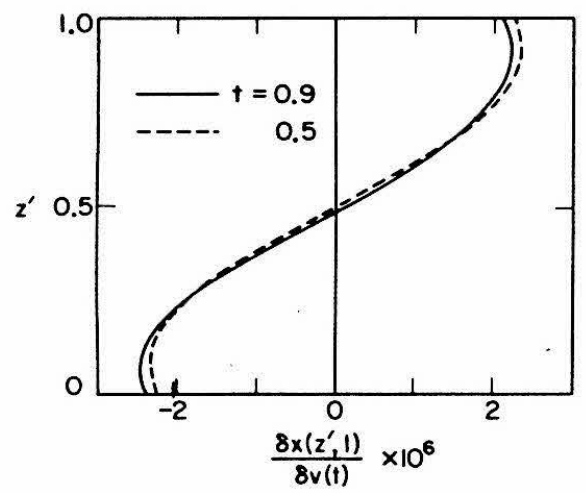

\section{REFERENCES}

[1] M. Koda, A. Dogru, and J. H. Seinfeld, "Sensitivity analysis of partial differential equations with application to reaction and diffusion processes," J. Comput. Phys., vol. 30. pp. 259-282, 1979 .

[2] J. L. Lions, Optimal Control of Systems Governed by Partial Differential Equations. New York: Springer-Verlag, 1971

[3] W. H. Ray and J. Szekely. Process Optimization with Applications in Metallurgy and Chemical Engineering. New York: Wiley, 1973.

Fig. 2. Functional derivative sensitivity coefficient $\delta x\left(z^{\prime}, 1\right) / \delta v(t)$ of the state at $t_{f}=1$ as a function of $z^{\prime}$ for two values of $t$. Case 1 ) boundary conditions.

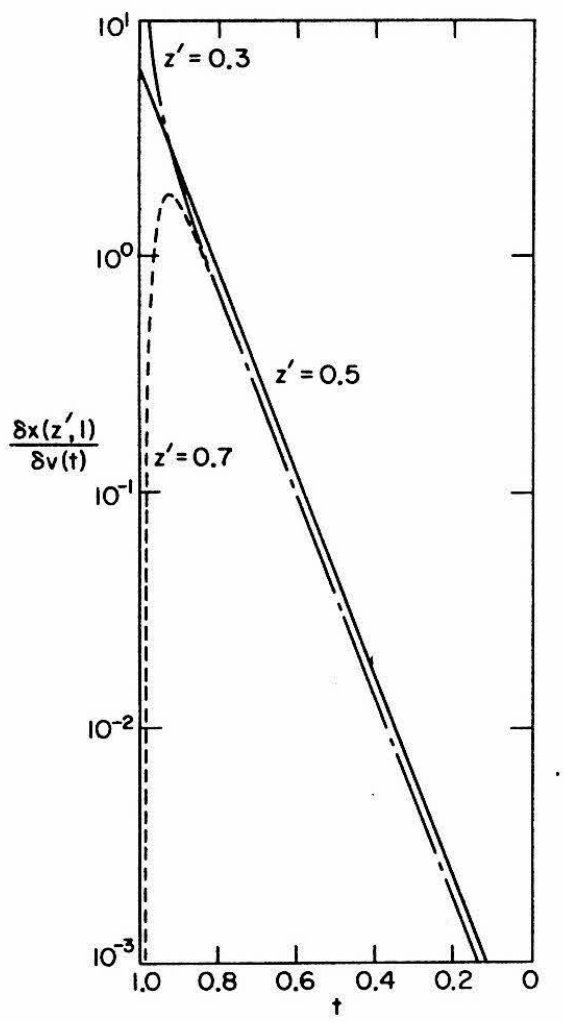

Fig. 3. Functional derivative sensitivity coefficient $\delta x\left(z^{\prime}, 1\right) / \delta v(t)$ of the state at $t_{f}=1$ as a function of $t$ for three values of $z^{\prime}$. Case 2) boundary conditions.

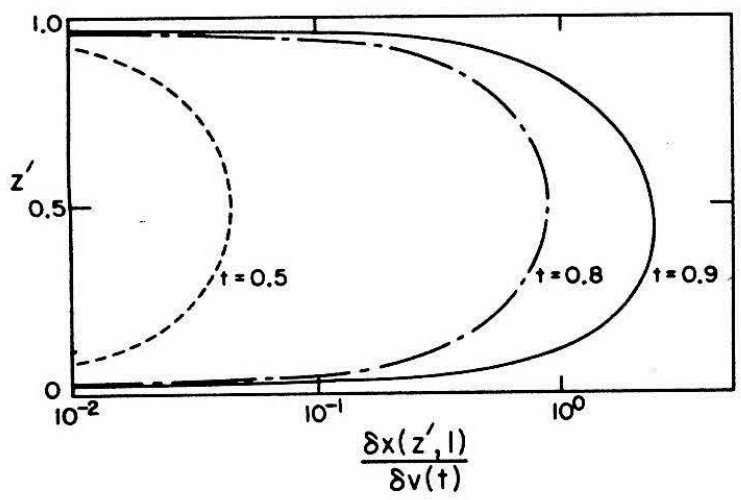

Fig. 4. Functional derivative sensitivity coefficient $\delta x\left(z^{\prime}, 1\right) / \delta v(t)$ of the state at $t_{f}=1$ as a function of $z^{\prime}$ for three values of $t$. Case 2) boundary conditions. 